\title{
A characterization of rational elements by Lüroth-type series expansions in the $p$-adic number field and in the field of Laurent series over a finite field
}

\author{
by \\ Vichian LAOHAKOSOL (Bangkok) and \\ NARAKORN ROMPURK (Khon Kaen)
}

1. Introduction. In a series of papers, A. Knopfmacher and J. Knopfmacher introduce general algorithms which lead to various unusual and unique infinite product ([1], [2]) or series expansions ([3], [4]) of $p$-adic numbers, as products or sums of rational numbers. From their work, the problem arises of characterizing rational numbers by such expansions. Of particular interest to us here is the problem of characterizing rational numbers by their series expansions. The Knopfmachers in [4] are able to establish a proposition which implies that two particular classes of their series expansions, namely, the Sylvester-type and Engel-type expansions are finite if and only if they represent rational numbers. At the end of [3], an open problem of characterizing the Lüroth-type representations for rational numbers is posed. We give such a characterization by proving that a $p$-adic number is rational if and only if its Lüroth series expansion is either finite or periodic. In addition, in the last section, the same result is also established for elements of the field of Laurent series over a finite field. The proof in the latter situation, though much like the one in the $p$-adic case, is simpler mainly due to the fact that there is no "carry" in the multiplication of Laurent series.

We first recall Knopfmachers' algorithm for constructing series expansion in the $p$-adic number field. Let $p$ be a fixed prime, $\mathbb{Q}_{p}$ the field of $p$-adic numbers equipped with the $p$-adic valuation $|\cdot|_{p}$, so normalized that $|p|_{p}$ $=p^{-1}$. For $A \in \mathbb{Q}_{p}$, define the order $\nu(A)$ of $A$ by $|A|_{p}=p^{-\nu(A)}$, and $\nu(0)=+\infty$. Each $A \in \mathbb{Q}_{p}$ is uniquely representable in the form

$$
A=\sum_{n=\nu(A)}^{\infty} c_{n} p^{n}, \quad c_{n} \in F_{p}:=\{0,1, \ldots, p-1\}, \quad c_{\nu(A)} \neq 0 .
$$

2000 Mathematics Subject Classification: 11A67, 11J61, 11J72. 
The fractional part, $\langle A\rangle$, of $A$ is defined as the finite series

$$
\langle A\rangle= \begin{cases}\sum_{n=\nu(A)}^{0} c_{n} p^{n} & \text { if } \nu(A) \leq 0, \\ 0 & \text { if } \nu(A)>0 .\end{cases}
$$

Denote the set of all fractional parts by

$$
S_{p}:=\left\{\langle A\rangle: A \in \mathbb{Q}_{p}\right\} \subset \mathbb{Q} .
$$

Knopfmachers' series expansion algorithm proceeds as follows: for $A \in \mathbb{Q}_{p}$, let $a_{0}:=\langle A\rangle \in S_{p}$. Define $A_{1}:=A-a_{0}$. If $A_{n} \neq 0(n \geq 1)$ is already defined, put

$$
a_{n}=\left\langle\frac{1}{A_{n}}\right\rangle \in S_{p}, \quad A_{n+1}=\left(A_{n}-\frac{1}{a_{n}}\right) \frac{s_{n}}{r_{n}}
$$

if $a_{n} \neq 0$, where $r_{n}, s_{n}$ are non-zero rational numbers which may depend on $a_{1}, \ldots, a_{n}$. Then

$$
\begin{aligned}
A & =a_{0}+A_{1}=a_{0}+\frac{1}{a_{1}}+\frac{r_{1}}{s_{1}} A_{2}=\cdots \\
& =a_{0}+\frac{1}{a_{1}}+\frac{r_{1}}{s_{1}} \frac{1}{a_{2}}+\cdots+\frac{r_{1} \cdots r_{n-1}}{s_{1} \cdots s_{n-1}} \frac{1}{a_{n}}+\frac{r_{1} \cdots r_{n}}{s_{1} \cdots s_{n}} A_{n+1} .
\end{aligned}
$$

The process ends in a finite expansion if some $A_{n+1}=0$. If some $a_{n}=0$, then $A_{n+1}$ is not defined. This is ruled out by imposing the condition

$$
\nu\left(s_{n}\right)-\nu\left(r_{n}\right) \geq 2 \nu\left(a_{n}\right)-1 .
$$

Thus

$$
A=a_{0}+\frac{1}{a_{1}}+\sum_{n=1}^{\infty} \frac{r_{1} \cdots r_{n}}{s_{1} \cdots s_{n}} \cdot \frac{1}{a_{n+1}}
$$

and this is called the Oppenheim-type expansion of $A$. Such a representation is said to be periodic if there are indices $m$ and $r$ such that $a_{n}=a_{n+r}, r_{n}=$ $r_{n+r}$ and $s_{n}=s_{n+r}$ for every $n \geq m$. We are interested here in the case where $r_{n}=1, s_{n}=a_{n}\left(a_{n}-1\right)(n \geq 1)$; then the algorithm yields a well defined (with respect to the $p$-adic valuation) and unique series expansion, termed the Lüroth-type expansion, of the form

$$
A=a_{0}+\frac{1}{a_{1}}+\sum_{n=1}^{\infty} \frac{1}{a_{1}\left(a_{1}-1\right) \cdots a_{n}\left(a_{n}-1\right) a_{n+1}} .
$$

From the construction, we also have the following two shapes for $A_{n}$, one from the head and the other for the tails: 


$$
\begin{aligned}
A_{n}= & A_{1} a_{1}\left(a_{1}-1\right) a_{2}\left(a_{2}-1\right) \cdots a_{n-1}\left(a_{n-1}-1\right) \\
& -\left(a_{1}-1\right) a_{2}\left(a_{2}-1\right) \cdots a_{n-1}\left(a_{n-1}-1\right) \\
& -\left(a_{2}-1\right) a_{3}\left(a_{3}-1\right) \cdots a_{n-1}\left(a_{n-1}-1\right)-\cdots \\
& -\left(a_{n-2}-1\right) a_{n-1}\left(a_{n-1}-1\right)-\left(a_{n-1}-1\right)
\end{aligned}
$$

and

$$
A_{n}=\frac{1}{a_{n}}+\frac{1}{a_{n}\left(a_{n}-1\right) a_{n+1}}+\frac{1}{a_{n}\left(a_{n}-1\right) a_{n+1}\left(a_{n+1}-1\right) a_{n+2}}+\cdots .
$$

Since $A_{n} \neq 0$, writing $1 / A_{n}=a_{n}+\sum_{r \geq 1} c_{r}^{\prime} p^{r}$, say, we get

$$
\nu\left(a_{n} A_{n}-1\right)=\nu\left(A_{n} \sum_{r \geq 1} c_{r}^{\prime} p^{r}\right) \geq \nu\left(A_{n}\right)+1
$$

and it is easily checked by induction for Lüroth-type expansions that $\nu\left(a_{n}\right)$ $\leq-1$ for $n \geq 1$ so that $\nu\left(r_{n}\right)=0$ and $\nu\left(s_{n}\right)=2 \nu\left(a_{n}\right)$. It is shown in Section 4 of [3] that periodic Lüroth-type expansions always represent rational numbers. Our first main result reads:

THEOREM 1. Let $S_{p}$ be the set of fractional parts defined above. A p-adic number $A$ whose Lüroth-type series expansion is

$$
A=a_{0}+\frac{1}{a_{1}}+\sum_{n=1}^{\infty} \frac{1}{a_{1}\left(a_{1}-1\right) \cdots a_{n}\left(a_{n}-1\right) a_{n+1}}
$$

with $a_{n} \in S_{p} \backslash\{0\}(n \geq 1)$ is rational if and only if the expansion is finite or periodic.

Let us recall from real analysis that a sequence of non-negative real numbers $\left(u_{n}\right)$ has no bounded subsequences if and only if its $n$th term tends to $\infty$ with $n$. In the proof of Theorem 1 , speaking about boundedness of any kind, we always refer to the usual absolute value.

2. Proof of Theorem 1. That finite or periodic Lüroth expansions always represent rational numbers is easily checked. It remains to show that each infinite Lüroth expansion which represents a rational must be periodic. In this case each $A_{n}(n \geq 1)$ is a non-zero rational number and so can be uniquely written in the form

$$
A_{n}=p^{\nu_{n}} \frac{\alpha_{n}}{\beta_{n}}
$$

where $\nu_{n}:=\nu\left(A_{n}\right), \alpha_{n} \in \mathbb{Z}, \beta_{n} \in \mathbb{N}, \operatorname{gcd}\left(\alpha_{n}, \beta_{n}\right)=1$ and $p \nmid \alpha_{n} \beta_{n}$. For $n \geq 1$, since $a_{n} \in S_{p} \backslash\{0\}$, we can uniquely write

$$
0<a_{n}=\frac{c_{\nu_{n}}}{p^{\nu_{n}}}+\cdots+\frac{c_{D_{n}}}{p^{D_{n}}}=\frac{c_{\nu_{n}}+\cdots+c_{D_{n}} p^{\nu_{n}-D_{n}}}{p^{\nu_{n}}}=: \frac{b_{n}}{p^{\nu_{n}}}<p
$$


where $c_{\nu_{n}}(\neq 0), \ldots, c_{D_{n}}(\neq 0) \in\{0,1, \ldots, p-1\},-\nu\left(a_{n}\right)=\nu\left(A_{n}\right)=\nu_{n} \geq$ $D_{n} \geq 0$ and $b_{n}=c_{\nu_{n}}+\cdots+c_{D_{n}} p^{\nu_{n}-D_{n}} \in \mathbb{N}$.

Rewriting

$$
A_{n+1}=\left(A_{n}-\frac{1}{a_{n}}\right) a_{n}\left(a_{n}-1\right)
$$

we get

$$
p^{\nu_{n+1}+\nu_{n}} \alpha_{n+1} \beta_{n}=\beta_{n+1}\left\{\alpha_{n} b_{n}-\beta_{n}\right\}\left(b_{n}-p^{\nu_{n}}\right) .
$$

Since $p \nmid \beta_{n+1}$ and $\operatorname{gcd}\left(\alpha_{n+1}, \beta_{n+1}\right)=1$, equation (2) implies that $\beta_{n+1} \mid \beta_{n}$. Thus there exist positive integers $N_{0}$ and $\beta$ such that $\beta_{n}=\beta$ for all $n \geq N_{0}$, and (2) becomes

$$
p^{\nu_{n+1}+\nu_{n}} \alpha_{n+1}=\left\{\alpha_{n} b_{n}-\beta\right\}\left(b_{n}-p^{\nu_{n}}\right) \quad\left(n \geq N_{0}\right) .
$$

Throughout this section, $|\cdot|$ denotes the usual absolute value. If there exists an infinite subsequence $\left\{n_{k}\right\} \subset \mathbb{N}$ such that the sequence $\left\{\left|\alpha_{n_{k}}\right| p^{\nu_{n_{k}}}\right\}_{k}$ is bounded, then the sequence $\left\{\alpha_{n_{k}} p^{\nu_{n_{k}}}\right\}_{k}$ is also bounded. As it is a bounded sequence of rational integers, there exist indices $i, j$ such that $N_{0} \leq i<j$ and

$$
\alpha_{n_{i}} p^{\nu_{n_{i}}}=\alpha_{n_{j}} p^{\nu_{n_{j}}}
$$

which yields

$$
A_{n_{i}}=\frac{\alpha_{n_{i}} p^{\nu_{n_{i}}}}{\beta}=\frac{\alpha_{n_{j}} p^{\nu_{n_{j}}}}{\beta}=A_{n_{j}}
$$

and so

$$
a_{n_{i}}=\left\langle\frac{1}{A_{n_{i}}}\right\rangle=\left\langle\frac{1}{A_{n_{j}}}\right\rangle=a_{n_{j}} .
$$

By (1), $A_{n_{i}+1}=A_{n_{j}+1}$, which implies that the expansion is ultimately periodic.

Henceforth, we assume that there exists a positive integer $N_{1}\left(\geq N_{0}\right)$ such that the sequence $\left\{\left|\alpha_{n}\right| p^{\nu_{n}}\right\}_{n \geq N_{1}}$ has no bounded subsequences. From this assumption, we aim to derive a contradiction in order to finish the proof.

To ease the writing, for $a_{n} \in S_{p}$, let $\bar{a}_{n}=a_{n}\left(a_{n}-1\right)$ and $\underline{a}_{n}=a_{n}-1$. Note that since $\left\{\left|\alpha_{n}\right| p^{\nu_{n}}\right\}_{n \geq N_{1}}$ has no bounded subsequences, there exists a positive integer $N_{2}\left(\geq N_{1}\right)$ such that

$$
\left|\alpha_{n}\right| p^{\nu_{n}} \geq 4 \beta \quad \text { whenever } n \geq N_{2} .
$$

We continue by verifying two important claims.

Claim 1. Let $a_{n}, c_{n} \in S_{p}, A_{n}$ as defined above, and $B_{n}:=A_{n} \bar{a}_{n}-\underline{a}_{n}$, $C_{n}:=A_{n} \bar{c}_{n}-\underline{c}_{n}$. For $n \geq N_{2}$, if $a_{n}>c_{n}>1$, then $\left|B_{n}\right|>\left|C_{n}\right|$.

To see this, observe that since $a_{n}>c_{n}>1$, if $A_{n}<0$, then both $B_{n}, C_{n}<0$ and

$$
B_{n}-C_{n}=\left\{A_{n}\left(a_{n}+c_{n}-1\right)-1\right\}\left(a_{n}-c_{n}\right)<0 .
$$


Thus $\left|B_{n}\right|>\left|C_{n}\right|$. While if $A_{n}>0$ (i.e., $\alpha_{n}>0$ ), then for $n \geq N_{2}$, we see that

$$
B_{n}=\left(a_{n}-1\right)\left(\frac{\alpha_{n} p^{\nu_{n}}}{\beta} a_{n}-1\right)>0,
$$

and similarly, $C_{n}>0$. Furthermore,

$$
\left|B_{n}\right|=B_{n}=\left(a_{n}-1\right)\left(A_{n} a_{n}-1\right)>\left(c_{n}-1\right)\left(A_{n} c_{n}-1\right)=C_{n}=\left|C_{n}\right| .
$$

Claim 2. For $n \geq N_{2}$, if $0<a_{n} \leq 1$, then $\left|A_{n+1}\right|<\left|A_{n}\right|$.

To see this, given $a_{n} \leq 1$, since $\nu_{n} \leq-1$, we have $a_{n}<1$ and so $D_{n}>0$. From

$$
1 \leq b_{n}:=c_{\nu_{n}}+\cdots+c_{D_{n}} p^{\nu_{n}-D_{n}} \leq p^{\nu_{n}-D_{n}+1}-1,
$$

we get

$$
1 \leq p^{\nu_{n}}-p^{\nu_{n}-D_{n}+1}+1 \leq p^{\nu_{n}}-b_{n} \leq p^{\nu_{n}}-1 .
$$

Writing $p^{\nu_{n}}-b_{n}=: d_{n} p^{\nu_{n}}$, then $d_{n} \in \mathbb{Q}$ and

$$
0<1-\frac{1}{p^{D_{n}-1}}+\frac{1}{p^{\nu_{n}}} \leq d_{n} \leq 1-\frac{1}{p^{\nu_{n}}}<1 .
$$

Substituting these values back into (3), we get

$$
p^{\nu_{n+1}} \alpha_{n+1}=\left(-\alpha_{n}\right) p^{\nu_{n}}\left(1-d_{n}\right) d_{n}+\beta d_{n} .
$$

Using the estimate $0<\left(1-d_{n}\right) d_{n} \leq 1 / 4$ and (4), when $n \geq N_{2}$ we have

$$
p^{\nu_{n+1}}\left|\alpha_{n+1}\right|<\frac{1}{4}\left|\alpha_{n}\right| p^{\nu_{n}}+\beta \leq \frac{1}{4}\left|\alpha_{n}\right| p^{\nu_{n}}+\frac{1}{4}\left|\alpha_{n}\right| p^{\nu_{n}}<\left|\alpha_{n}\right| p^{\nu_{n}},
$$

which yields

$$
\left|A_{n+1}\right|=p^{\nu_{n+1}} \frac{\left|\alpha_{n+1}\right|}{\beta}<p^{\nu_{n}} \frac{\left|\alpha_{n}\right|}{\beta}=\left|A_{n}\right| .
$$

From Claims 1 and 2, we see that starting from $n=N_{2}$ onwards, given $\left|A_{n}\right|$, the value of $\left|A_{n+1}\right|$ can only increase when $a_{n}>1$ and in this situation such increase is maximal when $a_{n}$ is as large as possible, i.e. as close to $p$ as possible.

We now consider the sequence $\mathbf{A}^{\prime}:=\left\{A_{N_{2}}^{\prime}, A_{N_{2}+1}^{\prime}, A_{N_{2}+2}^{\prime}, \ldots\right\}$, called the maximal sequence, which starts with $A_{N_{2}}^{\prime}=A_{N_{2}}$ and continues via the recurrence (1) but with the values of $a_{n}$ as large as possible, in particular, $a_{n} \approx p$. If we show that the maximal sequence $\mathbf{A}^{\prime}$ is bounded, then so also must be our original sequence $\left\{A_{n}\right\}$, which in turn implies that the sequence $\left\{\left|\alpha_{n}\right| p^{\nu_{n}}=\left|A_{n}\right| \beta\right\}_{n \geq N_{2} \geq N_{1}}$ is bounded, contradicting the assumption that it has no bounded subsequences, and the theorem will be proved.

To this end, we may assume that for $n \geq N_{2}$,

$$
p-\frac{3}{2}<p-\frac{1}{2}<a_{n}<p
$$


and so

$$
\left(p-\frac{3}{2}\right)^{2}<a_{n}\left(a_{n}-1\right)=\bar{a}_{n}<p(p-1) .
$$

From the expression for the tail

$$
A_{n}^{\prime}=\frac{1}{a_{n}}+\frac{1}{\bar{a}_{n} a_{n+1}}+\frac{1}{\bar{a}_{n} \bar{a}_{n+1} a_{n+2}}+\cdots \quad\left(n \geq N_{2}\right),
$$

note that the right-hand sum always converges $p$-adically but does not generally converge with respect to the usual absolute value. However, for the maximal sequence, it converges for both valuations and represents the same rational number; indeed,

$$
\begin{aligned}
\left|A_{n}^{\prime}\right|< & \frac{1}{p-3 / 2}+\frac{1}{(p-3 / 2)^{2}(p-3 / 2)} \\
& +\frac{1}{(p-3 / 2)^{2}(p-3 / 2)^{2}(p-3 / 2)}+\cdots \\
= & \frac{p-3 / 2}{(p-3 / 2)^{2}-1},
\end{aligned}
$$

which shows the boundedness of the maximal sequence as desired.

3. Case of formal series over a finite field. Let $\mathbb{F}_{q}$ be a finite field of order $q=p^{k}$ where $p$ is a prime and $k \in \mathbb{N}$ and let $p(x)$ be an irreducible polynomial of degree $d$ over $\mathbb{F}_{q}$. There are two main types of valuations on the field $\mathbb{F}_{q}(x)$ of rational functions: the $p(x)$-adic valuation, denoted by $\|\cdot\|_{p}$, and the infinite valuation, denoted $\|\cdot\|$. These two valuations are defined as follows: if $f(x) / g(x)=p(x)^{m} r(x) / s(x)$, where $f(x), g(x), r(x), s(x) \in$ $\mathbb{F}_{q}[x] \backslash\{0\}$ and $p(x) \nmid r(x) s(x)$, then

$$
\begin{aligned}
\|0\|_{p}=0, & \left\|\frac{f(x)}{g(x)}\right\|_{p}=q^{-m d} \\
\|0\|=0, & \left\|\frac{f(x)}{g(x)}\right\|=q^{\operatorname{deg} f(x)-\operatorname{deg} g(x)} .
\end{aligned}
$$

The valuation $\|\cdot\|$ can also be considered formally as a $1 / x$-adic valuation for the field $\mathbb{F}_{q}(1 / x)\left(=\mathbb{F}_{q}(x)\right)$.

Let $\mathbb{F}_{q}((p(x)))$ and $\mathbb{F}_{q}((1 / x))$ be the completions of $\mathbb{F}_{q}(x)$ with respect to the $p(x)$-adic and the infinite valuations, respectively. The extensions of the valuations to $\mathbb{F}_{q}((p(x)))$ and $\mathbb{F}_{q}((1 / x))$ will also be denoted by $\|\cdot\|_{p}$ and $\|\cdot\|$, respectively.

For $A \in \mathbb{F}_{q}((p(x)))$ (respectively, $B \in \mathbb{F}_{q}((1 / x))$ ), define the order $\nu(A)$ of $A$ (respectively, $\mu(B)$ of $B$ ) via $\|A\|_{p}=q^{-d \nu(A)}$ (respectively, $\|B\|=$ $\left.q^{-\mu(B)}\right)$ and set $\nu(0)=+\infty=\mu(0)$. 
It is well known that each $A \in \mathbb{F}_{q}((p(x))$ ) (respectively, each $B \in$ $\left.\mathbb{F}_{q}((1 / x))\right)$ is uniquely representable in the form

$$
A=\sum_{n=\nu(A)}^{\infty} b_{n}(x) p(x)^{n}, \quad b_{n}(x) \in \mathbb{F}_{q}[x], \operatorname{deg} b_{n}(x)<d, b_{\nu(A)}(x) \neq 0,
$$

respectively,

$$
B=\sum_{n=\mu(B)}^{\infty} c_{n}\left(\frac{1}{x}\right)^{n}, \quad c_{n} \in \mathbb{F}_{q}, c_{\mu(B)} \neq 0 .
$$

The fractional part, $\langle A\rangle_{p(x)}$, of $A$ (respectively, $\langle B\rangle_{1 / x}$ of $B$ ) is defined as the finite series

$$
\langle A\rangle_{p(x)}=\sum_{n=\nu(A)}^{0} b_{n}(x) p(x)^{n} \quad \text { if } \nu(A) \leq 0, \quad \text { and } 0 \text { otherwise }
$$

respectively,

$$
\langle B\rangle_{1 / x}=\sum_{n=\mu(B)}^{0} c_{n}(1 / x)^{n} \quad \text { if } \mu(B) \leq 0, \quad \text { and } 0 \text { otherwise. }
$$

Denote the sets of all fractional parts by

$$
S_{p(x)}:=\left\{\langle A\rangle_{p(x)}: A \in \mathbb{F}_{q}((p(x)))\right\} \subset \mathbb{F}_{q}(x),
$$

respectively,

$$
S_{1 / x}:=\left\{\langle B\rangle_{1 / x}: B \in \mathbb{F}_{q}((1 / x))\right\} \subset \mathbb{F}_{q}(x) .
$$

Knopfmachers' algorithm described in the introduction is also applicable in this setting and leads to analogous results. With similar notation, note that here also the inequality

$$
\left.\nu\left(s_{n}\right)-\nu\left(r_{n}\right) \geq 2 \nu\left(a_{n}\right)-1 \quad \text { (respectively, } \mu\left(s_{n}\right)-\mu\left(r_{n}\right) \geq 2 \mu\left(a_{n}\right)-1\right)
$$

is a sufficient condition for the expansion to exist in the field $\mathbb{F}_{q}((p(x)))$ (respectively, $\left.\mathbb{F}_{q}((1 / x))\right)$. Hence, $A \in \mathbb{F}_{q}((p(x)))$ (respectively, $B \in \mathbb{F}_{q}((1 / x))$ ) has an Oppenheim-type expansion of the form

$$
a_{0}+\frac{1}{a_{1}}+\sum_{n=1}^{\infty} \frac{r_{1} \cdots r_{n}}{s_{1} \cdots s_{n}} \cdot \frac{1}{a_{n+1}} .
$$

Such a representation is said to be periodic if there are indices $m$ and $r$ such that $a_{n}=a_{n+r}, r_{n}=r_{n+r}$, and $s_{n}=s_{n+r}$ for every $n \geq m$. For both fields of Laurent series, if we set $r_{n}=1$ and $s_{n}=a_{n}\left(a_{n}-1\right)$, then the algorithm yields a unique series expansion, called the Lüroth-type expansion, of the form

$$
a_{0}+\frac{1}{a_{1}}+\sum_{n=1}^{\infty} \frac{1}{a_{1}\left(a_{1}-1\right) \cdots a_{n}\left(a_{n}-1\right) a_{n+1}},
$$


and as in the case of $p$-adic numbers, $\nu\left(a_{n}\right) \leq-1$ (respectively, $\mu\left(a_{n}\right) \leq-1$ ) for all $n \geq 1$.

Our objective now is to prove that rational elements in these fields have either finite or periodic Lüroth-type expansions. Since the proofs for both fields are basically the same, we only provide the one for $\mathbb{F}_{q}((p(x)))=: \mathbf{F}_{q}^{p}$. The crux of the proof follows the same lines as in the $p$-adic case; however, since there is no "carry" in the multiplication of power series (or polynomials), the proof is not only simpler, but we can prove somewhat more.

THEOREM 2. Let $r_{n}=1$ and $s_{n}=a_{n} f\left(a_{n}\right)$ for some fixed $f(x) \in \mathbb{F}_{q}[x]$. If $\left\|a_{n}\right\| \leq 1$ for $n$ sufficiently large, then the corresponding Oppenheim-type expansion of $A \in \mathbf{F}_{q}^{p}$ is finite or ultimately periodic if and only if $A \in \mathbb{F}_{q}(x)$.

Proof. The fact that finite Oppenheim expansions yield rational elements is trivial, while that periodic expansions also give rational elements can be readily checked as in Section 4 of [3].

It remains to show that if the Oppenheim expansion of $A \in \mathbb{F}_{q}(x)$ is infinite, then it is ultimately periodic. Since each $A$ is rational, so is each $A_{n}(n \geq 1)$, which allows us to write it uniquely in the form

$$
A_{n}=p(x)^{\nu\left(A_{n}\right)} \frac{\alpha_{n}(x)}{\beta_{n}(x)}
$$

where $\alpha_{n}(x), \beta_{n}(x) \in \mathbb{F}_{q}[x]$ with $\operatorname{gcd}\left(\alpha_{n}(x), \beta_{n}(x)\right)=1, p(x) \nmid \alpha_{n}(x)$ and $p(x) \nmid \beta_{n}(x)$. For $n \geq 1$, since $a_{n} \in S_{p(x)} \backslash\{0\}$, we can write uniquely

$$
a_{n}=\frac{c_{\nu_{n}}(x)}{p(x)^{\nu_{n}}}+\cdots+\frac{c_{D_{n}}(x)}{p(x)^{D_{n}}}=\frac{c_{\nu_{n}}(x)+\cdots+c_{D_{n}}(x) p(x)^{\nu_{n}-D_{n}}}{p(x)^{\nu_{n}}}=\frac{b_{n}(x)}{p(x)^{\nu_{n}}}
$$

where $c_{\nu_{n}}(x)(\neq 0), \ldots, c_{D_{n}}(x)(\neq 0) \in S_{p(x)}, \nu_{n}:=\nu\left(A_{n}\right)=-\nu\left(a_{n}\right) \geq D_{n} \geq 0$ and

$$
b_{n}(x)=c_{\nu_{n}}(x)+\cdots+c_{D_{n}}(x) p(x)^{\nu_{n}-D_{n}} \in \mathbb{F}_{q}[x] \backslash\{0\} .
$$

It is easy to see that

$$
\left\|a_{n}\right\|=q^{-d D_{n}+\operatorname{deg} c_{D_{n}}(x)}
$$

and

$$
\left\|b_{n}(x)\right\|=q^{\operatorname{deg} c_{D_{n}}(x)+d \nu_{n}-d D_{n}} \leq q^{d-1+d \nu_{n}-d D_{n}} .
$$

Let $s_{n}=a_{n} f\left(a_{n}\right):=a_{n}\left(f_{m} a_{n}^{m}+\cdots+f_{1} a_{n}+f_{0}\right) \in a_{n} \mathbb{F}_{q}\left[a_{n}\right]$. Rewriting

$$
A_{n+1}=\left(A_{n}-\frac{1}{a_{n}}\right) \frac{s_{n}}{r_{n}}
$$

we get

$$
\times\left\{f_{m} b_{n}(x)^{m}+f_{m-1} b_{n}(x)^{m-1} p(x)^{\nu_{n}}+\cdots+f_{1} b_{n}(x) p(x)^{(m-1) \nu_{n}}+f_{0} p(x)^{m \nu_{n}}\right\} .
$$


Since $p(x) \nmid \beta_{n+1}(x)$ and $\operatorname{gcd}\left(\alpha_{n+1}(x), \beta_{n+1}(x)\right)=1$, equation (8) implies that $\beta_{n+1}(x) \mid \beta_{n}(x)$, which yields $0 \leq \operatorname{deg} \beta_{n+1}(x) \leq \operatorname{deg} \beta_{n}(x)(n \geq 1)$. Thus there exist a positive integer $N_{0}$ and a non-negative integer $M$ such that $\operatorname{deg} \beta_{n}(x)=M$ for all $n \geq N_{0}$, i.e.,

$$
\left\{\operatorname{deg} \beta_{n}(x)\right\} \text { is a finite set }
$$

and

$$
\beta_{n}(x)=c_{n} \beta_{N_{0}}(x), \quad c_{n} \in \mathbb{F}_{q} \backslash\{0\} \quad\left(n \geq N_{0}\right) .
$$

Since $\nu_{n} \geq 1$ and $p(x) \nmid \beta_{n+1}(x)$ and $p(x) \nmid b_{n}(x)$, equation (8) also implies that for $n \geq N_{0}$,

$$
p(x)^{\nu_{n+1}+m \nu_{n}} \mid\left\{\alpha_{n}(x) b_{n}(x)-c_{n} \beta_{N_{0}}(x)\right\}
$$

and so

$$
d \nu_{n+1}+m d \nu_{n} \leq \max \left\{\operatorname{deg} \alpha_{n}(x)+\operatorname{deg} b_{n}(x), M\right\} .
$$

If there exists an infinite subsequence $\left(n_{k}\right) \subset \mathbb{N}$ with all $n_{k} \geq N_{0}$ such that

$$
\operatorname{deg} \alpha_{n_{k}}(x)+\operatorname{deg} b_{n_{k}}(x) \leq M,
$$

then the two sequences of non-negative integers $\left\{\operatorname{deg} \alpha_{n_{k}}(x)+\operatorname{deg} b_{n_{k}}(x)\right\}$ and $\left\{\operatorname{deg} \alpha_{n_{k}}(x)\right\}$ are bounded, and from (11) so is the sequence $\left(\nu_{n_{k}}\right)$. We deduce from the unique representation $A_{n_{k}}=p(x)^{\nu_{n_{k}}} \alpha_{n_{k}}(x) / \beta_{n_{k}}(x)$ that $\left\{A_{n_{k}}\right\}$ is a finite set. Hence, there exist indices $K<L$ such that all $A_{n_{K}}=$ $A_{n_{L}}$ and so $a_{n_{K}}=\left\langle 1 / A_{n_{K}}\right\rangle=\left\langle 1 / A_{n_{L}}\right\rangle=a_{n_{L}}$ and $s_{n_{K}}=s_{n_{L}}$. Invoking (7), we see that $A_{n_{K}+1}=A_{n_{L}+1}$ and so the expansion is ultimately periodic.

Henceforth, assume that there exists a positive integer $N_{1} \geq N_{0}$ such that

$$
\operatorname{deg} \alpha_{n}(x)+\operatorname{deg} b_{n}(x)>M \quad\left(n \geq N_{1}\right) .
$$

Going back to equation (8) and using (10), for $n \geq N_{1}$ we get

$$
\begin{aligned}
& p(x)^{\nu_{n+1}+m \nu_{n}} \alpha_{n+1}(x) c_{n}=c_{n+1}\left\{\alpha_{n}(x) b_{n}(x)-c_{n} \beta_{N_{0}}(x)\right\} \\
& \times\left\{f_{m} b_{n}(x)^{m}+f_{m-1} b_{n}(x)^{m-1} p(x)^{\nu_{n}}+\cdots+f_{1} b_{n}(x) p(x)^{(m-1) \nu_{n}}+f_{0} p(x)^{m \nu_{n}}\right\} .
\end{aligned}
$$

Taking infinite valuation, we get

$$
\begin{aligned}
& q^{d \nu_{n+1}+m d \nu_{n}+\operatorname{deg} \alpha_{n+1}(x)} \leq \max \left\{\left\|\alpha_{n}(x)\right\|\left\|b_{n}(x)\right\|, q^{M}\right\} \\
& \quad \times \max \left\{\left\|b_{n}(x)\right\|^{m},\left\|b_{n}(x)\right\|^{m-1} q^{d \nu_{n}}, \ldots,\left\|b_{n}(x)\right\| q^{(m-1) d \nu_{n}}, q^{m d \nu_{n}}\right\} \\
& =\left\|\alpha_{n}(x)\right\|\left\|b_{n}(x)\right\| \\
& \quad \times \max \left\{\left\|b_{n}(x)\right\|^{m},\left\|b_{n}(x)\right\|^{m-1} q^{d \nu_{n}}, \ldots,\left\|b_{n}(x)\right\| q^{(m-1) d \nu_{n}}, q^{m d \nu_{n}}\right\}
\end{aligned}
$$

using (12). From the given hypothesis, we may assume, without loss of generality, that $\left\|a_{n}\right\| \leq 1$ when $n \geq N_{1}$. We distinguish two possible cases. 
CASE 1: $\left\|a_{n}\right\|<1$. In this case, $D_{n}>0$, yielding $d \nu_{n}-d D_{n}+d-1<d \nu_{n}$ and $\left\|b_{n}(x)\right\|<q^{d \nu_{n}}$. Relation (13) becomes

$$
q^{d \nu_{n+1}+m d \nu_{n}+\operatorname{deg} \alpha_{n+1}(x)}<q^{\operatorname{deg} \alpha_{n}(x)+d \nu_{n}+m d \nu_{n}},
$$

i.e., we have the strict inequality

$$
d \nu_{n+1}+\operatorname{deg} \alpha_{n+1}(x)<d \nu_{n}+\operatorname{deg} \alpha_{n}(x) .
$$

CASE 2: $\left\|a_{n}\right\|=1$. In this case, $D_{n}=0$ and $c_{D_{n}}(x)=: c \in \mathbb{F}_{q} \backslash\{0\}$, which yields $\left\|b_{n}(x)\right\|=q^{d \nu_{n}}$. Relation (13) thus implies

$$
\begin{aligned}
& q^{d \nu_{n+1}+m d \nu_{n}+\operatorname{deg} \alpha_{n+1}(x)} \\
& \leq q^{\operatorname{deg} \alpha_{n}(x)+d \nu_{n}} \max \left\{q^{m d \nu_{n}}, q^{(m-1) d \nu_{n}+d \nu_{n}}, \ldots, q^{d \nu_{n}+(m-1) d \nu_{n}}, q^{m d \nu_{n}}\right\} \\
& =q^{\operatorname{deg} \alpha_{n}(x)+d \nu_{n}+m d \nu_{n}}
\end{aligned}
$$

i.e., we have the non-strict inequality

$$
d \nu_{n+1}+\operatorname{deg} \alpha_{n+1}(x) \leq d \nu_{n}+\operatorname{deg} \alpha_{n}(x) .
$$

From both cases, we see that $\left\{d \nu_{n}+\operatorname{deg} \alpha_{n}(x)\right\}_{n \geq N_{1}}$ is a non-increasing sequence of positive integers. Hence both $\left\{\nu_{n}\right\}_{n \geq N_{1}}$ and $\left\{\operatorname{deg} \alpha_{n}(x)\right\}_{n \geq N_{1}}$ are bounded sets of non-negative integers. Consequently, there are infinitely many $n \geq N_{1}$ such that all $\nu_{n}$ are equal and all $\operatorname{deg} \alpha_{n}(x)$ are the same. Taking into account (10), we deduce that there are $M>L>N_{1}$ such that $A_{M}=A_{L}$. Invoking (7), we conclude that the expansion is ultimately periodic.

The condition $\left\|a_{n}\right\| \leq 1$ in Theorem 2 can be removed by taking $p(x)=x$, and we get

Corollary 3. For $r_{n}=1$ and $s_{n}=a_{n} f\left(a_{n}\right)$ for some fixed $f(x) \in$ $\mathbb{F}_{q}[x]$, the Oppenheim-type expansion of $A \in \mathbb{F}_{q}((x))$ is finite or ultimately periodic if and only if $A \in \mathbb{F}_{q}(x)$.

Specializing $f(x)=x-1$ in Theorem 2 , we get a characterization of rational elements by their Lüroth expansions.

COROLlaRY 4. If $\left\|a_{n}\right\| \leq 1$, then the corresponding Lüroth-type expansion of $A \in \mathbf{F}_{q}^{p}$ is finite or ultimately periodic if and only if $A \in \mathbb{F}_{q}(x)$.

COROLlary 5. The Lüroth-type expansion of $A \in \mathbb{F}_{q}((x))$ is finite or ultimately periodic if and only if $A \in \mathbb{F}_{q}(x)$.

Finally, as described earlier, an analogous proof yields the same result in $\mathbb{F}_{q}((1 / x))$, and we mention in particular:

Theorem 6. In $\mathbb{F}_{q}((1 / x))$, the Lüroth-type expansion of $A$ is finite or ultimately periodic if and only if $A \in \mathbb{F}_{q}(x)$.

Acknowledgements. We wish to thank the referee and the copy editor for their helpful comments. 


\section{References}

[1] A. Knopfmacher and J. Knopfmacher, A product expansion in p-adic and other nonArchimedean fields, Proc. Amer. Math. Soc. 104 (1988), 1031-1035.

[2] - - - $p$-adic and non-Archimedean product representations, Results Math. 15 (1989), $324-334$.

[3] - - - Series expansions in p-adic and other non-Archimedean fields, J. Number Theory 32 (1989), 297-306.

[4] - - - Infinite series expansions for p-adic numbers, ibid. 41 (1992), 131-145.

Department of Mathematics

Kasetsart University

Bangkok 10900, Thailand

E-mail: fscivil@ku.ac.th.

Department of Mathematics Khon Kaen University Khon Kaen 40002, Thailand E-mail: rompurk@hotmail.com

Received on 5.10.2005

and in revised form on 8.2.2006 